\title{
Evaluation of Clipped-OFDM and SC/FDE Alternatives for Block Transmission Using Iterative Receiver Techniques
}

\author{
Paulo Torres \\ IPCB-EST, Polyt. Inst. of Castelo Branco, Portugal, \\ Email: paulotorres@est.ipcb.pt
}

\author{
António Gusmão \\ CAPS-IST, Tech. Univ. of Lisbon, Portugal, \\ Email: gus@ist.utl.pt
}

\begin{abstract}
A CP-assisted (Cyclic Prefix) block transmission is widely accepted as a good choice for future mobile systems, taking advantage of low-cost, flexible, FFT-based (Fast Fourier Transform) signal processing technology with both OFDM (Orthogonal Frequency Division Multiplexing) and SC (Single Carrier) alternatives. The OFDM alternative provides a simple receiver implementation which allows good performances, by exploiting the channel coding effort, but is known to suffer from a high PMEPR (Peak-to-Mean Envelope Power Ratio), leading to power amplification difficulties. The SC/FDE (Frequency-Domain Equalization) alternative is easily compatible with low PMEPR values, but the FDE (MMSE) receiver performance is known to be far from the optimum.

In this paper, we consider a reduced-PMEPR, ClippedOFDM transmission, where an advanced receiver performs the iterative cancellation of the deliberate nonlinear distortion effects. In parallel, we consider an advanced Turbo FDE receiver, as well as simplified iterative FDE receiver implementations, within the SC alternative. The "SC vs OFDM" comparisons of this paper, under the use of iterative receiver techniques in both cases, show a very significant overall advantage for the SC side.
\end{abstract}

\section{INTRODUCTION}

The wireless access of future mobile broadband systems will likely follow, in many cases, a CP-assisted (Cyclic Prefix) block transmission approach, employing either OFDM (Orthogonal Frequency Division Multiplexing) or SC (Single Carrier) modulation schemes [1]. Mixed CPassisted solutions have already been proposed, with OFDM for the downlink and SC for the uplink [2], [3], [4]. Within current implementations, the $\mathrm{CP}$ is long enough to cope with the maximum relative channel delay. Therefore, in what concerns the useful part of each received burst, IBIfree conditions are ensured and the linear convolutions inherent to the time-dispersive channels become equivalent to circular convolutions in the time domain (i.e. multiplications in the frequency domain). This paves the way for the use of low-cost, FFT-based techniques to compensate for the severe channel distortion effects.

OFDM schemes are known to suffer from strong envelope fluctuations, requiring a quasi-linear power amplification and significant power backoffs [5]. One approach to reduce the PMEPR (Peak-to-Mean Envelope Power Ratio) at the amplifier input involves a deliberate nonlinear distortion through a digital signal processing method: this is the case of schemes which combine the required nonlinear operation (e.g., of the clipping type) in the time domain with a frequency-domain filtering, so as to keep the spectral efficiency of ordinary OFDM [6]. In a very recent paper [7], the ideas of [8] on iterative cancelation of nonlinear distortion effects, at the receiver side, were extended to the classes of signal processing schemes for OFDM-type transmission proposed in [6], under a full-length CP.

On the other hand, conventional SC modulations with a reduced PMEPR can easily be designed. Under a full-length CP,a low-complexity FDE (Frequency-Domain Equalization) technique, involving simple FFT computations, can be employed to solve the severe ISI problem. Recently, the possibility of achieving improved FDE performances in SC-based systems, under full-length $\mathrm{CP}$ conditions, was demonstrated [9]. In the meantime, an alternative approach for Turbo FDE implementation, which is able to deal with reduced-CP conditions, was also developed [10].

In this paper, we consider a reduced-PMEPR, ClippedOFDM transmission where a modified version of the iterative receiver technique described in [7] is employed. In parallel, we consider the Turbo FDE receiver approach of [10], as well as simplified iterative FDE receiver techniques, within the SC alternative. A number of numerical performance results is provided, so as to allow the appropriate "SC vs OFDM" comparisons.

\section{ITERATIVE RECEIVER TECHNIQUES FOR SC/FDE BLOCK TRANSMISSION}

When a full-length CP is employed, Turbo FDE techniques such as that proposed in [9] can provide a strongly improved FDE performance, while avoiding a high complexity of implementation. Such techniques, which resort to a conventional, linear, single-tap FDE scheme, take advantage of the SISO decoder outputs in order to carry out a turbo soft-cancelation of residual ISI through the use of the soft information on the coded bits. According to the "switched APPLE/MF approach" (APProximate Linear (MMSE) Equalization/Matched Filtering) proposed in [9], the FDE coefficients for each iteration are selected on the basis of an "average SNR" concerning the length- $N$ block of equalizer outputs. This block plays the role of an extrinsic information on the coded data block, delivered by the equalizer to the channel SISO decoder. For each iteration, the receiver uses the algorithm (APPLE (MMSE) or MF) leading to the largest estimated SNR.

In [10] we described a new "conventional" (for fulllength CP conditions) Turbo FDE technique which is 
strongly related to that proposed in [9], but replaces the selection principle (APPLE/MF) regarding the linear FDE parameters by an appropriate "compromise choice": in fact, the values of the linear FDE parameters are adaptively adjusted, iteration by iteration, according to the available block of SISO decoder outputs. The proposed receiver structure is as depicted in Fig. 1 ( $\Pi$ and $\Pi^{-1}$ stand for "interleaver" and "deinterleaver", respectively, and $\odot$ denotes "element-by-element multiplication".). At the equalizer output, the time-domain vector $\tilde{\mathbf{s}}^{(i)}(m)$ is the IDFT of

$$
\tilde{\mathbf{S}}^{(i)}(m)=\mathbf{F}^{(i)}(m) \odot \mathbf{Y}(m)+\mathbf{G}^{(i)}(m) .
$$

Therefore, the $N$ entries of $\tilde{\mathbf{S}}^{(i)}(m)$ can be written as

$$
\tilde{S}_{k}^{(i)}(m)=F_{k}^{(i)}(m) Y_{k}(m)+G_{k}^{(i)}(m)
$$

where $F_{k}^{(i)}(m), k=0,1, \ldots, N-1$, are the multiplicative FDE parameters for iteration $i$, and

$$
G_{k}^{(i)}(m)=\left(\gamma^{(i)}(m)-F_{k}^{(i)}(m) \hat{H}_{k}\right) \bar{S}_{k}^{(i-1)}(m),
$$

with $\gamma^{(i)}(m)=(1 / N) \sum_{k=0}^{N-1} F_{k}^{(i)}(m) \hat{H}_{k}$ are complementary FDE parameters for ISI soft-cancelation purposes. $\overline{\mathbf{S}}^{(i-1)}(m)=\left[\bar{S}_{0}^{(i-1)}(m), \bar{S}_{1}^{(i-1)}(m), \ldots, \bar{S}_{N-1}^{(i-1)}(m)\right]^{T}$ is the DFT of $\overline{\mathbf{s}}^{(i-1)}(m)=\left[\bar{s}_{0}^{(i-1)}(m)\right.$, $\left.\bar{s}_{1}^{(i-1)}(m), \ldots, \bar{s}_{N-1}^{(i-1)}(m)\right]^{T}$, resulting from the soft information provided by the SISO decoder as shown in Appendix A, when assuming a QPSK (Quaternary Phase Shift Keying) modulation. It should be noted that, in this case,

$$
\begin{array}{r}
\bar{s}_{p}^{(i-1)}(m)=\frac{\sigma_{s}}{\sqrt{2}} . \\
\left(\tanh \left(\frac{L_{p, I}^{(i-1)}(m)}{2}\right)+j \tanh \left(\frac{L_{p, Q}^{(i-1)}(m)}{2}\right)\right),
\end{array}
$$

where $L_{p, I}^{(i-1)}(m)$ and $L_{p, Q}^{(i-1)}(m)$ are the LLRs of the "inphase coded bit" and "quadrature coded bit", respectively ( $\sigma_{s}^{2}$ is the variance of the time-domain QPSK symbol coefficients)

The soft demapper in Fig. 1 provides the inputs to the SISO decoder (LLRs of the several coded bits). The decoder outputs must correspond to the full soft information, not the extrinsic one. As to the FDE parameters, alternatively to $F_{k}^{(i)}(m)=\hat{H}_{k}^{*} /\left(\hat{\alpha}+\left|\hat{H}_{k}\right|^{2}\right)$ (APPLE) or $F_{k}^{(i)}(m)=\hat{H}_{k}^{*}$ (MF) [9], with $\alpha=\frac{\sigma_{n}^{2}}{\sigma^{2}}\left(\sigma_{n}^{2}\right.$ denoting the variance of the Gaussian noise components), we adopt

$$
F_{k}^{(i)}(m)=\frac{K_{F}^{(i)}(m) \hat{H}_{k}^{*}}{\hat{\alpha}+\left(1-\left(\hat{\rho}^{(i-1)}(m)\right)^{2}\right)\left|\hat{H}_{k}\right|^{2}},
$$

where $\hat{\rho}^{(i-1)}(m)=\frac{1}{N} \sum_{n=0}^{N-1} \frac{E\left[s_{n}^{*}(m) \hat{s}_{n}^{(i-1)}(m)\right]}{E\left[\left|s_{n}(m)\right|^{2}\right]}$ is an "overall correlation coefficient" [10]. It can be obtained as an average value of the $2 N$ correlation coefficients per bit $\left(\rho_{n, I}^{(i-1)}(m), \rho_{n, Q}^{(i-1)}(m)\right)$, derived from the SISO decoder outputs. $K_{F}^{(i)}(m)$ is a normalization factor: using $\gamma^{(i)}(m)=1, \tilde{s}_{n}^{(i)}(m)=s_{n}(m)+\xi_{n}^{(i)}(m)$, where $\xi_{n}^{(i)}(m)$ is the zero-mean "error" (assumed to be approximately complex Gaussian) concerning symbol $s_{n}(m)$ at the FDE output. Under the "Gaussian assumption", the LLRs of the "in-phase bit" and the "quadrature bit", at the SISO decoder input, are given by $L_{n, I}^{(i)}(m)=\frac{\sqrt{8}}{\sigma_{e q}^{(i)}(m)} \sigma_{s} \operatorname{Re}\left\{\tilde{s}_{n}^{(i)}(m)\right\}$ and $L_{n, Q}^{(i)}(m)=\frac{\sqrt{8}}{\sigma_{e q}^{2(i)}(m)} \sigma_{s} \operatorname{Im}\left\{\tilde{s}_{n}^{(i)}(m)\right\}$ respectively, where $\sigma_{e q}^{2(i)}(m)$ is the mean-squared error in the time-domain samples $\tilde{s}_{n}^{(i)}(m)$. It can easily be estimated as $\hat{\sigma}_{e q}^{(i)}(m)=$ $\frac{1}{N} \sum_{n=0}^{N-1}\left|\tilde{s}_{n}^{(i)}(m)-s_{n}^{\prime(i)}(m)\right|^{2}$, with $s_{n}^{\prime(i)}(m)=$ $\frac{\sigma_{s}}{\sqrt{2}}\left(\operatorname{sgn}\left(\operatorname{Re}\left\{\tilde{s}_{n}^{(i)}(m)\right\}\right)+j \operatorname{sgn}\left(\operatorname{Im}\left\{\tilde{s}_{n}^{(i)}(m)\right\}\right)\right)$.

We point out that, for $i=1$, the $F_{k}^{(i)}(m)$ parameters meet the MMSE criterion, since $\hat{\rho}^{(i-1)}(m)=0$ in (5). After a number of iterations and/or for high SNRs, typically $\hat{\rho}_{n, I}^{(i-1)}(m) \approx 1$ and $\hat{\rho}_{n, Q}^{(i-1)}(m) \approx 1$, leading to $\hat{\rho}^{(i-1)}(m) \approx 1$ in $(5)$, and, therefore, to $F_{k}^{(i)}(m)$ parameters approximately in accordance with the MF criterion. When replacing the "APPLE/MF selection" [9] of the $F_{k}^{(i)}(m)$ parameters by the proposed "compromise choice" (eq. (5)), a small performance gain is achieved [10].

Simplified iterative FDE implementations, based on the ideas above, can be considered, with no decoding effort really involved in the FDE process [10]. The corresponding receiver derives from that shown in Fig. 1, by suppressing the SISO decoder; therefore, no extrinsic information is provided to help the iterative FDE process, and the soft FDE outputs in a given iteration $(i-1)$ are directly used to compute $F_{k}^{(i)}(m)$ and $G_{k}^{(i)}(m)$ for next iteration. In this paper, we consider this type of simplified iterative FDE technique, followed by a conventional Viterbi decoding. We also consider here another simplified iterative FDE technique, as follows:

- The SISO decoder in Fig. 1 is replaced by a conventional Viterbi decoder, which means that the soft estimates $\bar{s}_{n}^{(i-1)}(m)$ are replaced by conventional hard estimates $\hat{s}_{n}^{(i-1)}(m)$, when computing the equalization parameters $G_{k}^{(i)}(m)$;

- The equalization parameters $F_{k}^{(i)}(m)$ are computed according to the selection principle (APPLE/MF), as in [9].

\section{ITERATIVE RECEIVER TECHNIQUES FOR OFDM BLOCK TRANSMISSION UNDER A Deliberate Nonlinear Transmission}

\section{A. Nonlinear Signal Processing Scheme for Generating Reduced-PMEPR OFDM-Type Signals}

The nonlinear signal processing scheme considered here has been described in detail in [6].

The modified length-N blocks $\mathbf{S}^{C F}(m)=$ $\left[S_{0}^{C F}(m), \cdots, S_{N-1}^{C F}(m)\right]^{T}$ are obtained from the conventional blocks $\mathbf{S}(m)=\left[S_{0}(m), \cdots, S_{N-1}(m)\right]^{T}$ as shown in fig. A, through a nonlinear (NL), clipping operation in the time domain, followed 


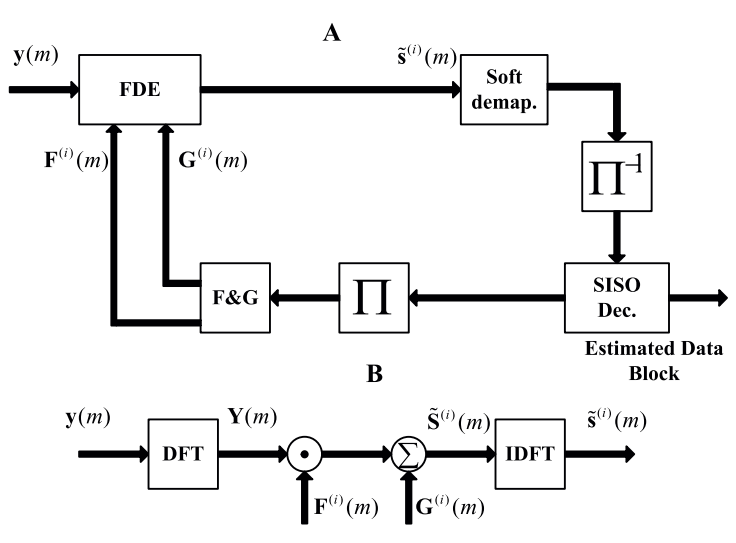

Fig. 1. Turbo FDE receiver structure (A) and characterization of the FDE unit (B) for SC-based block transmission.

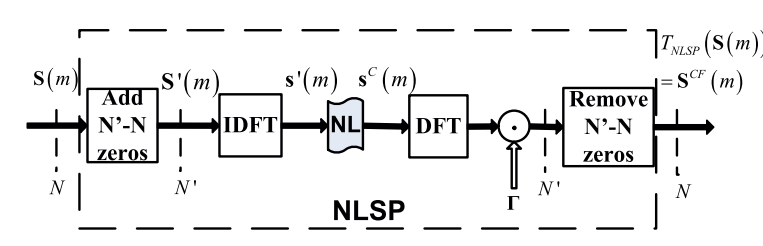

Fig. 2. Nonlinear signal processing scheme (NLSP) to reduce the PMEPR of OFDM signals, while preserving the spectral efficiency of ordinary OFDM.

by a frequency-domain filtering. It is assumed here that $s_{n}^{C}(m)=f\left(\left|s_{n}^{\prime}(m)\right|\right) \exp \left(j \arg \left(s_{n}^{\prime}(m)\right)\right)$, with $f\left(\left|s_{n}^{\prime}(m)\right|\right)=\left|s_{n}^{\prime}(m)\right|$ for $\left|s_{n}^{\prime}(m)\right|<s_{M}$ and $f\left(\left|s_{n}^{\prime}(m)\right|\right)=s_{M}$ for $\left|s_{n}^{\prime}(m)\right| \geq s_{M}$ (clipping level $s_{M}$ ). We also assume that $N^{\prime} / N=2$ and the complementary frequency-domain filtering is characterized by $\Gamma_{k}=0$ or 1 , in the "out-of-band" region or the "in-band" region, respectively. The clipping level can be chosen for a selected PMEPR reduction, and the subsequent frequencydomain filtering cancels the out-of-band distortion effects of the nonlinearity (producing, however, some regrowth of the envelope fluctuations).

Whenever $N>>1$, both $\Re e\left\{s_{n}^{\prime}(m)\right\}$ and $\Im m\left\{s_{n}^{\prime}(m)\right\}$ are approximately Gaussian, with zero mean and a given variance $\sigma^{2}$. It can be shown that the transmitted frequencydomain samples can be decomposed into two uncorrelated terms, as follows:

$$
S_{k}^{C F}(m)=\beta S_{k}(m)+D_{k}(m),
$$

where, according to the clipping level $s_{M}$,

$$
\beta=1-\exp \left(-\frac{k_{M}^{2}}{2}\right)+\frac{1}{2} \sqrt{2 \pi} k_{M} Q\left(k_{M}\right)
$$

for $k_{M}=\frac{s_{M}}{\sigma}$ and $D_{k}(m)$ exhibits quasi-Gaussian characteristics for any $k$ under a "high $N$ " assumption $\left(Q\left(k_{M}\right)=\frac{1}{\sqrt{2 \pi}} \int_{k_{M}}^{+\infty} \exp \left(-x^{2} / 2\right) d x\right)$.

\section{B. Iterative Cancelation of the Deliberate Nonlinear Distortion Effects}

When employing the nonlinear processing scheme described above at the OFDM transmitter, a certain BER per- formance degradation is unavoidable with a conventional OFDM receiver: this degradation can be rather small for very small clipping efforts $\left(s_{M} / \sigma>3.5\right)$, but becomes significant when very low PMEPR values are intended $\left(s_{M} / \sigma<1.5\right)$.

In the conventional OFDM receiver, after guard removal, each block of received samples $\mathbf{y}(m)$ is converted into a frequency-domain block $\mathbf{Y}(m)=$ $\left[Y_{0}(m), \cdots, Y_{N-1}(m)\right]^{T}$ by a DFT processor. Thanks to the cyclic prefix, $Y_{k}(m)=S_{k}^{C F}(m) H_{k}+N_{k}(m)$, where $N_{k}(m)$ and $H_{k}$ concern the Gaussian noise and the overall channel response, respectively, at the $k$ th sub-channel. Having in mind (6),

$$
Y_{k}(m)=\beta S_{k}(m) H_{k}+D_{k}(m) H_{k}+N_{k}(m),
$$

for the $\mathrm{N}$ values of $k$. The BER performance degradation when using the deliberate nonlinear distortion is jointly due to the fact that a "useless" power is spent as a transmitted self-interference (see (6)) and to the fact that the received quasi-Gaussian self-interference on each subcarrier is added to the Gaussian channel noise.

The more sophisticated OFDM receiver considered in [7] could not avoid a certain performance degradation due to the useless transmitted power. However, it was able to reduce, through a DF-based (Decision Feedback) iterative cancelation procedure, the negative impact of the selfinterference term in (8).

In this paper, we consider a similar receiver structure. However, a soft cancellation is adopted here, by employing a SISO decoder and by considering the reliability of the coded bit decisions, so as to mitigate the error propagation. This turbo-cancellation of nonlinear distortion effects is performed within a receiver structure as depicted in Fig. 3. The components of vector $\mathbf{F}^{(i)}(m)$ are given by $F_{k}=$ $\frac{1}{\beta \hat{H}_{k}}(k=0,1, \cdots, N-1)$, while

$\mathbf{G}^{(i)}(m)=\hat{\rho}^{(i-1)}(m)\left[\hat{\mathbf{S}}^{(i-1)}(m)-\frac{1}{\beta} T_{N L S P}\left(\hat{\mathbf{S}}^{(i-1)}(m)\right)\right]$,

where $\hat{\rho}^{(i-1)}(m)$ is an average value of the $2 N$ correlation coefficients per bit, $\rho_{k, I}^{(i-1)}(m)$ and $\rho_{k, Q}^{(i-1)}(m)$, derived from the SISO decoder outputs (see Appendix B). A QPSK symbol constellation is assumed, as in the SC case.

It should be noted that $\mathbf{Y}(m) \cdot \mathbf{F}=\mathbf{S}(m)+\frac{\mathbf{D}(m)}{\beta}+$ $\mathbf{N}(m)$, where $\frac{\mathbf{D}(m)}{\beta}=\frac{1}{\beta} T_{N L S P}(\mathbf{S}(m))-\mathbf{S}(m)$. By adding $\mathbf{G}^{(i)}(m)$ to $\mathbf{Y}(m) \cdot \mathbf{F}$, we are simply subtracting an estimate of $\frac{\mathbf{D}(m)}{\beta}$, for soft cancellation of the nonlinear distortion. Due to the nonlinear nature of the $T_{N L S P}($. operation, it is not appropriate to use $\overline{\mathbf{S}}^{(i-1)}(m)$ directly when computing $\mathbf{G}^{(i)}(m)$; the use of (9) is a realistic choice for creating the intended soft cancellation effect.

For SISO decoding purposes, the soft demapping of $\tilde{S}_{k}^{(i)}(m)$ gives the LLRs of the I and Q bits:

$$
L_{k, I}^{(i)}(m)=\frac{\sqrt{8}}{\hat{\sigma_{k}^{2}}} \sigma_{S} \Re e\left\{\tilde{S}_{k}^{(i)}(m)\right\}
$$


and

$$
L_{k, Q}^{(i)}(m)=\frac{\sqrt{8}}{\hat{\sigma_{k}^{2}}} \sigma_{S} \Im m\left\{\tilde{S}_{k}^{(i)}(m)\right\}
$$

where

$$
\hat{\sigma}_{k}^{2}=\frac{1}{\beta^{2}} \frac{E\left[\left|N_{k}(m)\right|^{2}\right]}{\left|\hat{H}_{k}\right|^{2}}
$$

( $\sigma_{S}^{2}$ denotes the variance of the frequency-domain symbol coefficients).

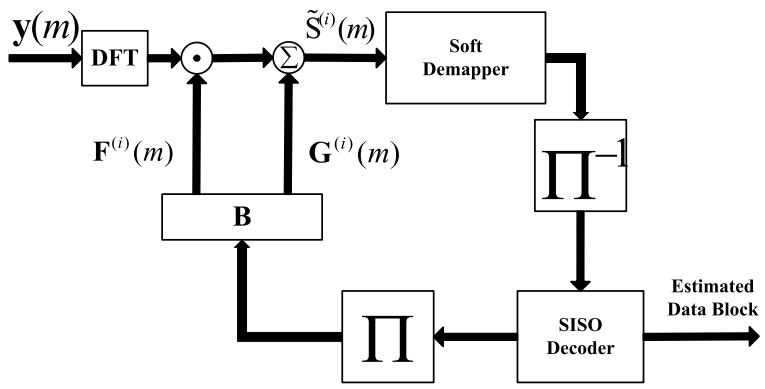

Fig. 3. Receiver structure for iterative cacellation of deliberate nonlinear distortion effects is OFDM-based block transmission.

\section{Performance Evaluation And SC/OFDM COMPARISONS}

In the following, we present a set of numerical performance results, with regards to broadband transmission over a strongly frequency-selective Rayleigh fading channel, when the iterative receiver techniques of Secs II and III are employed (power delay profile type $\mathrm{C}$ within HIPERLAN/2). A conventional CP-assisted block transmission is assumed, with $N=256$ QPSK data symbols per block, either defined in the time domain (SC/FDE alternative) or in the frequency domain (OFDM alternative). In both cases, we selected a 16-state rate-1/2 convolutional code with $G(D)=\left[\begin{array}{ll}1 & \frac{1+D^{2}+D^{3}+D^{4}}{1+D+D^{4}}\end{array}\right]$ and a low-complexity SISO decoding through the use of the Max-Log-MAP algorithm [11]. The duration of the useful part of each block is $5 \mu \mathrm{s}$, and a full-length CP $(\mathrm{L}=64)$ is adopted.

Fig. 4 shows BER performance results for the SC/FDE alternative, when employing the Turbo FDE technique described in Sec. II (solid lines), involving the contribution of a SISO decoder to the iterative equalization process, as well as the simplified iterative FDE implementations also proposed in Sec. II: the iterative FDE without decoding aid, followed by a conventional Viterbi decoding (dashed lines (a)); the iterative FDE using conventional Viterbi decoding (dot-dashed lines (b)). These results show that the latter simplification leads to a small performance degradation (less than $0.5 \mathrm{~dB}$ ), when compared with the SISO-based Turbo FDE. The other simplification ("out of loop" Viterbi decoding following an iterative FDE process without decoding aid), which clearly has a reduced complexity, leads to an increased degradation, exceeding $1.5 \mathrm{~dB}$, but preserves a performance gain exceeding 1.5
$\mathrm{dB}$ over the conventional SC/FDE receiver technique (first iteration).

In Fig. 5 we show BER performances for the clipped OFDM alternative, when employing the iterative receiver technique presented in Sec. III. This figure includes lower bounds on the BER performance, which have been obtaining by simulation, when supressing the $D_{k}(m) H_{k}$ term in (8). With a small clipping effort $\left(k_{M}=2.0\right)$, there is no significant performance gain through the use of the iterative technique, since the nonlinear distortion effects to the canceled are quite limited. However, for a strong clipping effort $\left(k_{M}=0.5\right)$, an increased performance gain can be achievable, and it should be noted that two iterations are enough to approximate that gain. Additionally, it can be noted that the BER performance with $k_{M}=0.5$ is less than $1.5 \mathrm{~dB}$ worse than the BER performance with $k_{M}=2$. Having in mind that the PMEPR values which correspond to $k_{M}=2.0$ and $k_{M}=0.5$ are $5.7 \mathrm{~dB}$ and $4.1 \mathrm{~dB}$, respectively, the overall power advantage of using $k_{M}=0.5$ instead of $k_{M}=2.0$ (implying 1.6dB of PMEPR reduction), when the iterative receiver technique of Sec. III is available, is even more clear.

Figs 6, 7 and 8 are concerned to "SC vs OFDM" comparisons. In Fig. 6, we compare BER performances with conventional (non-iterative) receiver techniques; this figure was obtained by selecting the appropriate performance curves (first iteration) from Figs 4 and 5. A small performance advantage (less than $0.5 \mathrm{~dB}$ ) for the OFDM side, when $k_{M}=2.0$, can be observed; however, the corresponding PMEPR value is $3 \mathrm{~dB}$ above the PMEPR value for the $\mathrm{SC}$ alternative $(2.7 \mathrm{~dB})$. In Fig. 7, also joining some performance curves from Figs 4 and 5, we compare BER performances when employing the iterative receiver techniques of Sec. II (SC/FDE alternative) and Sec. III (OFDM alternative): a significant performance advantage for the SC side is then observed, in terms of "BER against $E_{b} / N_{0}$ " (even the lowest-complexity, simplified iterative SC/FDE technique (a) gives better performance than the clipped-OFDM alternative). Fig. 8 includes the PMEPR issue in the overall "SC vs OFDM" comparisons, when using the iterative receiver techniques of Secs II and III. In this figure, we plot "BER against $\frac{E_{B}^{(p)}}{N_{0}}(d B)=$ $\frac{E_{B}}{N_{0}}(d B)+P M E P R(d B)$ " (as, for example, in [3]). The overall power-efficiency advantage for the SC side is even more significant, approximating $6 \mathrm{~dB}$ for the optimized Turbo FDE technique and exceeding $4 \mathrm{~dB}$ under the lowestcomplexity, simplified iterative SC/FDE technique.

\section{Conclusions}

In this paper, we considered two alternatives for reducedPMEPR block transmission using iterative receiver techniques. For the 'clipped OFDM' alternative, we considered an advanced receiver which performs an iterative cancellation of the nonlinear distortion effects. For the 'SC/FDE alternative', we considered an advanced Turbo FDE receiver technique, as well as simplified iterative 


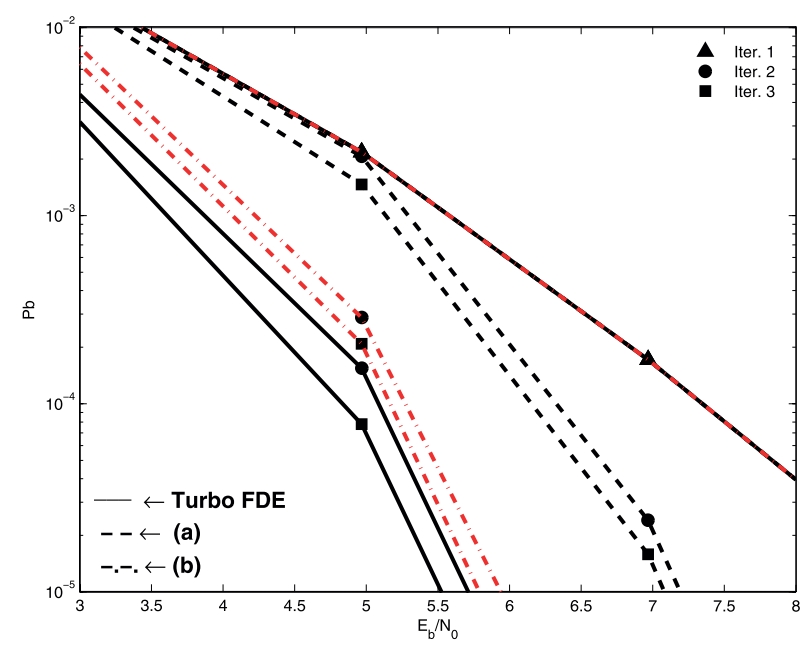

Fig. 4. Coded BER performances for the SC/FDE alternative, with either Turbo FDE (solid lines) or simplified (a) and (b) iterative FDE techniques.

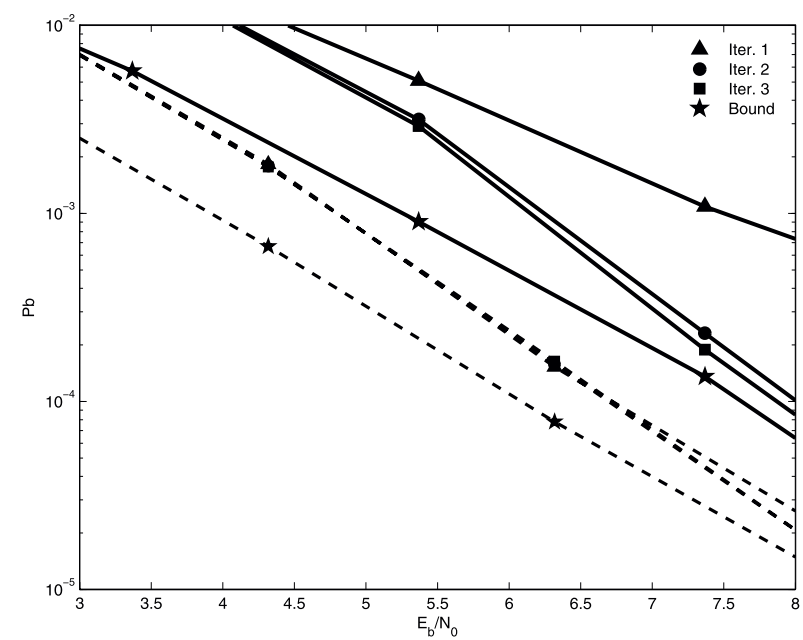

Fig. 5. Comparison of coded BER performances for clipped OFDM, with $k_{M}=0.5$ (solid lines) or $k_{M}=2.0$ (dashed lines).

FDE receiver techniques. The "SC vs OFDM" comparisons, involving a rate-1/2 convolutional code in both cases, show a very clear overall advantage for the SC side, much more significant than the advantage, already reported in the literature, concerning conventional (noniterative) receiver implementations [2], [3], [4]. It should be noted that there is a clear performance advantage for the SC side even when the lowest-complexity, simplified iterative FDE technique is employed. It should also be noted that the overall SC/FDE advantage is not due to any drawback of the iterative receiver technique for clipped OFDM: in fact, as shown in Fig. 5, the lower bound on the BER performance can be closely approximated with a few iterations when that technique is adopted. The SC/FDE advantage essentially derives from the fact that the energy of each symbol is distributed across the entire transmission

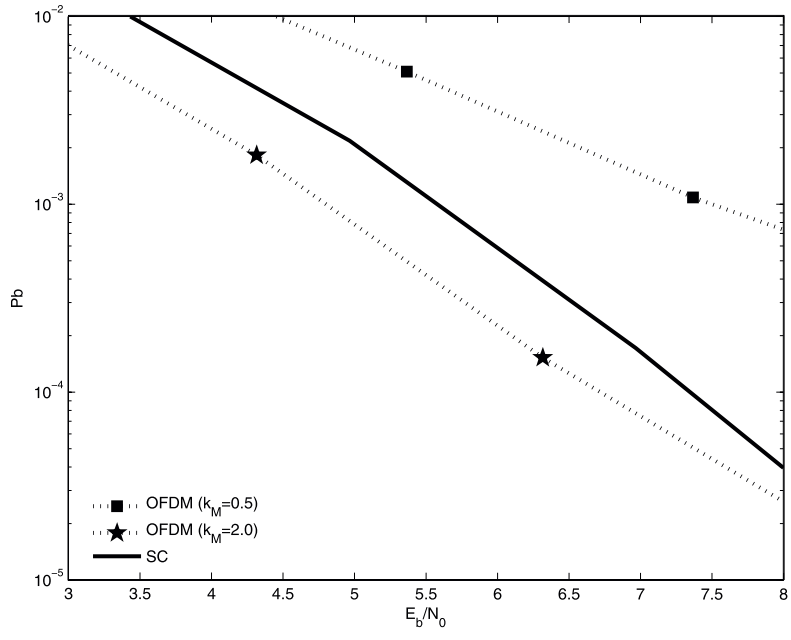

Fig. 6. Comparison of coded BER performances for clipped OFDM (with $k_{M}=0.5$ or $k_{M}=2.0$ ) and SC/FDE, with conventional receiver techniques.

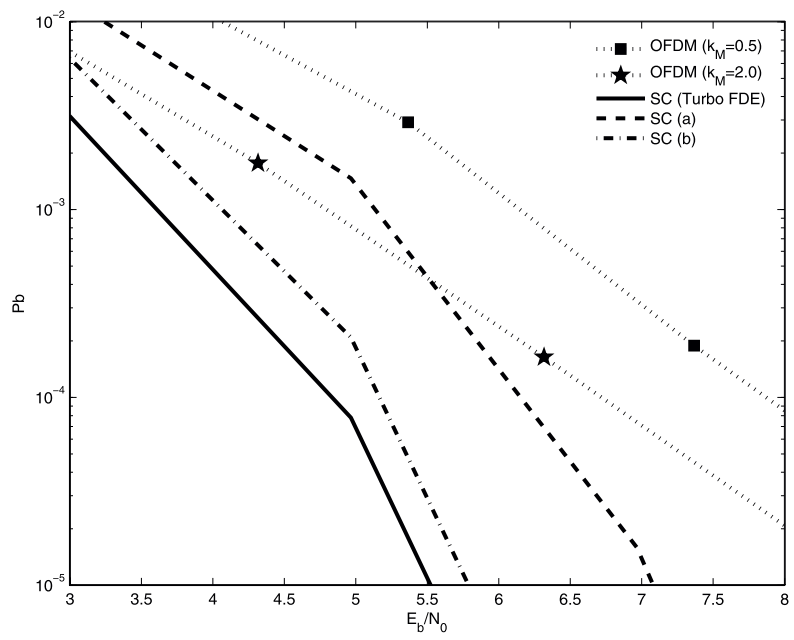

Fig. 7. Comparison of coded BER performances (3 iterations) for clipped OFDM, with $k_{M}=0.5$ or $k_{M}=2.0$ (dotted lines), and SC (Turbo FDE and simplified ((a) and (b)) iterative FDE).

bandwidth, together with the reduced PMEPR which is inherent to the SC modulation format.

\section{APPENDIX A}

\section{QPSK Symbol STATISTICS Using SofT Decoder} OUTPUTS IN THE SC CASE

Let us assume QPSK symbol coefficients $s_{n}=s_{n, I}+$ $j s_{n, Q}$, with $s_{n, I}= \pm \sigma_{s} / \sqrt{2}$ and $s_{n, Q}= \pm \sigma_{s} / \sqrt{2}$ ( $n=0,1, \ldots, N-1$ ), according to the coded data block. When the LLRs (log-likelihood ratios) concerning the $n$th in-phase bit and the $n$th quadrature bit, as provided by the channel decoder, are $L_{n, I}$ and $L_{n, Q}$, respectively, the resulting expected value $\bar{s}_{n}$ can be expressed as $\bar{s}_{n}=$ $\bar{s}_{n, I}+j \bar{s}_{n, Q}$ with $\bar{s}_{n, I}=\frac{\sigma_{s}}{\sqrt{2}} \tanh \left(\frac{L_{n, I}}{2}\right)$ and $\bar{s}_{n, Q}=$ $\frac{\sigma_{s}}{\sqrt{2}} \tanh \left(\frac{L_{n, Q}}{2}\right)$. 


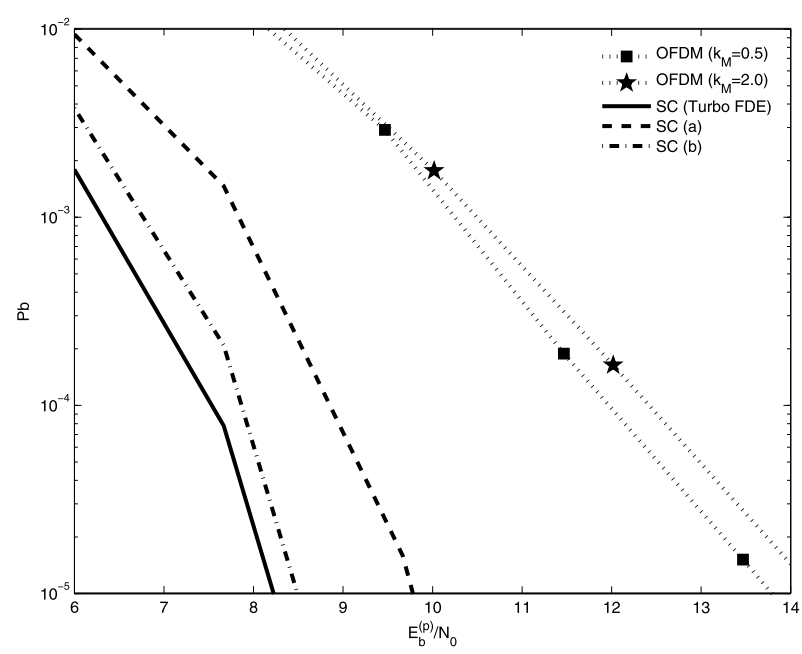

Fig. 8. Comparison of coded BER performances ( 3 iterations) for clipped OFDM, with $k_{M}=0.5$ or $k_{M}=2.0$ (dotted lines) and SC (Turbo FDE and simplified ((a) and (b)) iterative FDE), in terms of "BER against $E_{b}^{(p)} / N_{0} "$.

Let us define the "coded bit decisions", $\hat{s}_{n, I}= \pm \sigma_{s} / \sqrt{2}$ and $\hat{s}_{n, Q}= \pm \sigma_{s} / \sqrt{2}$, according to the signs of $L_{n, I}$ and $L_{n, Q}$, respectively, and the following correlation coefficients: $\rho_{n, I}=\frac{E\left[s_{n, I} \hat{s}_{n, I}\right]}{E\left[\left|s_{n, I}\right|^{2}\right]} ; \quad \rho_{n, Q}=\frac{E\left[s_{n, Q} \hat{s}_{n, Q}\right]}{E\left[\left|s_{n, Q}\right|^{2}\right]}$. Since $\rho_{n, I}=1-2 \operatorname{Prob}\left(\hat{s}_{n, I}=-s_{n, I} \mid L_{n, I}\right)=\tanh \left(\left|L_{n, I}\right| / 2\right)$ and $\rho_{n, Q}=1-2 \operatorname{Prob}\left(\hat{s}_{n, Q}=-s_{n, Q} \mid L_{n, Q}\right)=$ $\tanh \left(\left|L_{n, Q}\right| / 2\right)$ (leading to $0 \leq \rho_{n, I} \leq 1$ and $0 \leq \rho_{n, Q} \leq$ 1 ), the average values $\bar{s}_{n, I}$ and $\bar{s}_{n, Q}$ can be written as follows: $\bar{s}_{n, I}=\rho_{n, I} \hat{s}_{n, I} ; \quad \bar{s}_{n, Q}=\rho_{n, Q} \hat{s}_{n, Q}$.

\section{APPENDIX B \\ QPSK SYMbol STATISTICS USING Soft Decoder OUTPUTS IN THE OFDM CASE}

Let us assume QPSK symbol coefficients $S_{k}=S_{k, I}+$ $j S_{k, Q}$ in the frequency domain, with $S_{k, I}= \pm \sigma_{S} / \sqrt{2}$ and $S_{k, Q}= \pm \sigma_{S} / \sqrt{2}(k=0,1, \ldots, N-1)$, according to the coded data block. When the LLRs concerning the kth in-phase bit and the kth quadrature bit, as provided by the channel decoder, are $L_{k, I}$ and $L_{k, Q}$, respectively, the resulting expected value $\bar{S}_{k}$ is $\bar{S}_{k}=\bar{S}_{k, I}+j \bar{S}_{k, Q}$, with $\bar{S}_{k, I}=\frac{\sigma_{S}}{\sqrt{2}} \tanh \left(\frac{L_{k, I}}{2}\right)$ and $\bar{S}_{k, Q}=\frac{\sigma_{S}}{\sqrt{2}} \tanh \left(\frac{L_{k, Q}}{2}\right)$. As for the SC case, we can define "coded bit decisions" and "correlation coefficients" for both in-phase and quadrature coded bits. Obviously, $\bar{S}_{k, I}=\rho_{k, I} \hat{S}_{k, I}$ and $\bar{S}_{k, Q}=$ $\rho_{k, Q} \hat{S}_{k, Q}$.

\section{REFERENCES}

[1] H. Sari, G. Karam, and I. Jeanclaude, "An Analysis of Orthogonal Frequency-division Multiplexing for Mobile Radio Applications," Proc. IEEE VTC'94, June 1994.

[2] A. Gusmão, R. Dinis, J. Conceição, and N. Esteves, "Comparison of Two Modulation Choices for Broadband Wireless Communications," IEEE VTC'2000 (Spring), May 2000.

[3] P. Montezuma and A. Gusmão, "A Pragmatic Coded Modulation Choice for Future Broadband Wireless Communications," IEEE VTC'01 (Spring), May 2001.
[4] D. Falconer, S. Ariyavisitakul, A. Benyamin-Seeyar, and B. Eidson, "Frequency Domain Equalization for Single-Carrier Broadband Wireless Systems," IEEE Comm. Mag., vol. 4, no. 4, Apr. 2002.

[5] R. van Nee and R. Prasad, "OFDM for Wireless Multimedia Communications," Artech House Publications, 2000.

[6] R. Dinis and A. Gusmão, "A Class of Nonlinear Signal Processing Schemes for Bandwidth-Efficient OFDM Transmission with Low Envelope Fluctuation," IEEE Trans. on Comm., vol. 52, no. 11, Nov. 2004.

[7] A. Gusmão, R. Dinis, and P. Torres, "Low-PMEPR OFDM Transmission with an Iterative Receiver Technique for Cancellation of Nonlinear Distortion," IEEE VTC'05 (Fall), Sept. 2005.

[8] J. Tellado, L. Hoo, and J. Cioffi, "Maximum Likelihood Detection of Nonlinearly Distorted Multicarrier Symbols by Iterative Decoding," IEEE Trans. on Comm., vol. 51, Feb. 2003.

[9] M. Tüchler and J.Hagenauer, "Linear Time and Frequency Domain Turbo Equalization," IEEE VTC'01(Spring), May 2001.

[10] A. Gusmão, P. Torres, R. Dinis, and N. Esteves, "A Class of Iterative FDE Techniques for Reduced-CP SC-Based Block Transmission," IEEE ISTC'2006, Apr. 2006.

[11] B.Vucetic and J.Yuan, "Turbo Codes: Principles and Applications," Kluwer Academic Publ., 2002. 